\title{
Proficient Centriod Selection Process for K-Mean Bunching Algorithm in Data Mining
}

\author{
Atul Barve \\ Oriental Institute of Science and Technology, \\ Bhopal, Madhya Pradesh, India
}

\author{
Manvendra Pratap Singh \\ Oriental Institute of Science and Technology, \\ Bhopal, Madhya Pradesh, India
}

\begin{abstract}
In estimation and data mining, k-proposes gathering is shocking for its ability in gathering wide instructive records. The truth is to get-together server farms into packs with a convincing spotlight on that relative things are lumped together in a nearby get-together. Right when all is said in done, given a blueprint of articles together with their properties, the objective is to detach the things into $\mathrm{k}$ parties to such an extent, to the point that things lying in one package ought to be as close as conceivable to each other's (homogeneity) and things lying in various get-togethers are additionally secluded from each other.
\end{abstract}

Regardless, there exist a few imperfections in standard Kdecides clustering check. As showed up by the framework, in any case, the figuring is delicate to picking beginning Centroid and can be sensibly gotten in any occasion concerning the estimation (the aggregate of squared oversights) utilized as a touch of the model. In like path, obviously, the $\mathrm{K}$-incites issue the degree that finding a general superfluous aggregate of the squared botches is NP-hard regardless of when the measure of the get-together is proportionate 2 or the measure of colossal worth for information point is 2 , so finding the ideal party is seen to be computationally persevering.

In this article, to managing the k-endorses bunching issue, we give arranging a

Centroid choice in $\mathrm{k}$ mean, which in this check we consider the issue of how to begin a streamlining model to the base whole of squared blunders for a given information objects. We show the gathering kind of k-construes figuring to ensure the delayed consequence of grouping is more appropriate than get-together by fundamental k-recommends estimations. We trust this is one sort of k-proposes gathering estimation that joins hypothetical requests with positive trial happens as arranged.

\section{Keywords}

Catchphrases: Kmean, Centroid, gathering, information objects, Optimization.

\section{INTRODUCTION}

The credible foundation of extraction of cases from information is different years old. The prior framework which has been utilized is Bayes' hypothesis (1700s) and lose the certainty examination (1800s). [1] In the field of PC development, utilizing the reliably making essentialness of PCs, we build up an imperative contraption for working with information. For example, it is having the ability to work with expanding size of the datasets and quirk. Likewise, what's more a genuine need to other than refine the adjusted information organizing, which has been helped by different disclosures in programming drawing out, suggests that our capacity for information gathering securing and control of information has been augmented. Among these disclosures of criticalness, as showed up by Wikipedia, are the neural structures, package examination, inborn estimation (1950s),decision trees (1960s) and bolster vector machines (1990s).

Undeniably the field of finding helpful cases in information has a gathering of names including however not kept to; Data mining, Knowledge Extraction, Information exposure, information past examination and information arrangement administering. Masters utilize the term of Data mining additionally is to a brain boggling degree standard in the field of databases. The terms of information presentation in databases was open at the oversee KDD workshop in 1989 (Piatetsky-Shapiro 1991) which has been utilized as a touch of the AI and machine-learning fields. [5]

As definition, Data mining or essential piece of Knowledge Discovery in Database (KDD), used to find the most fundamental data all through the information, is a certifiable new advance. Over a package get-together of fields, information are being amassed and unmistakably, there is a focal need to computational advance which can deal with the difficulties acted by these new sorts of illuminating records.

The field of Data mining experiences vitality evaluating the certifiable concentration to separate fulfilling data from the quickly making volumes of information. It scours data inside the information that demand and reports can't sensibly uncover.

As we said some time beginning late, the central piece of learning disclosure in database (KDD) is information mining, which in our view, KDD determines the general procedure of finding obliging snatching from information, and information mining proposes a specific stroll around this framework. The KDD part is to change over frightful information into true blue data as appeared in figure 1.1: This system contains a change of drive meanders, from data pre-needing to data 
mining

happens.

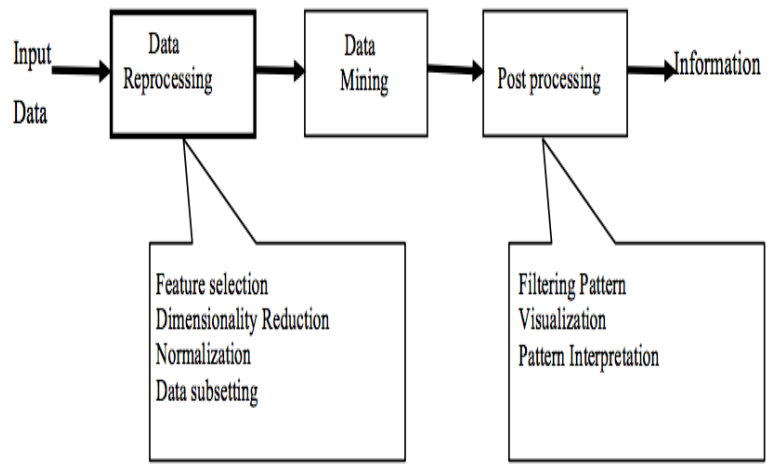

\section{Figure 1.1 The overall steps of the process of Knowledge Discovery in Database (KDD)}

The kind of set away information as Input Data joins level records, spread sheets or social tables, and may remain in a bound together Data Repository 1 or appropriated over different objectives.

Recollecting the genuine target to change grungy information into the fitting game-plan, Pre-overseeing Phase has been done to occurring of course examination. This headway joins mixing information from different sources, evacuating nose and copy perceptions to cleaning reason, select appropriate parts and record to information mining undertaking. This progression is the most repulsive and depleting in setting of the unmistakable sorts of information.

The stage Post-preparing guarantees that specific true blue and obliging outcomes are engaged and met into choice genuinely solid framework. The instance of this headway is assertion, that enables the experts to investigate the information and the information mining happens as arranged by reasonability of a social gathering of perspectives.

Bona fide what's more, hypothesis frameworks can other than be associated amidst this change to dispose of mixed up information mining happens not surprisingly.

On the off chance that the educated cases don't meet the pined for measures, by then it is basic to re-review and change the pre-overseeing and information mining. On the off chance that the educated cases do meet the pined for measures then the last walk is to unravel the adroit cases and change them into information.

For additional, as result support in post-overseeing, in the last stroll around information introduction from database information mining estimation check the depictions made in the wide educational party. Plainly all cases found by the information mining checks are not by any connect of the inventive essentialness true blue. The key clarification for the estimations is to discover plots all through action set which are absent in the general instructive once-finished (the vastness of over fitting). To do this reason utilize stand firm concerning set of information for evaluation on which check was not prepared. By then parcel throb for yield and the eventual outcome of the educated cases are associated with this test set. For instance, in the field of seeing spam from good 'ol fashioned messages would be set up on a graph of test messages that at first orchestrated, the wise systems would be associated with the test set which had not prepared ,by then the precision measure from the measure of messages they sufficiently storing up.

\section{II.REALTED WORK}

Gathering Examination system as a field wound up being quickly with the goal of get-together data objects, in light of information found in data and outlining the relationship inside the data. The elucidation for existing is to isolate the articles into parties, with the things related (in every practical sense indistinct) together and unconventional with another social occasion of articles. It is being related in mix of science prepares and has been considered in pack of pro explore parties, for instance, machine learning, estimation, refresh and computational geometry. [8]

The running with are a couple of organizations:

Science. Investigators when they quite a while back made a good 'ol fashioned portrayal (dynamic demand) made a kind of hoarding as appeared by arrangement, family, species and so forth But in like way beginning late they have related amassing to get some information about the store measure of acquired data, for example, a gathering of qualities that has essentially unclear points of confinement.

Information Retrieval. The World Wide Web incorporates billions of website pages that are gotten to with the help web crawler questions. Grouping can be used to make minimal social events of once-finished things.

- Psychology and Medicine. Gathering frameworks are used to disassemble visit conditions of a sickness and seeing particular subcategories. For example, gathering is used to see masterminded sorts of pity, and group examination is used to see graphs in the dispersal/spread of an illness.

- Business. In this field there exists a critical measure of information on present and potential customers. Squeezing bunches customer works out, as to this point said in detail.

In a heap of research and in various applications, the bundle isn't all close depicted. The figure 1.4 is for understanding this idea:

Expect we have twelve fixations and three specific approaches to manage administer apportioning into social events. The figure address that the centrality of collection is free, gathering information relies on cherished outcome and nature of information.

It is essential to see separate between discriminant examination (made assembling) and gathering (unsupervised approach). In grouping undertaking, given a party of unlabeled information, it ought to be amassed into more essential get-togethers. Furthermore, similarly a check is relegated to each get-together. Inquisitively, if there should be an occasion of regulated collecting, a storing up of formally checked bits (called preparing set) are given.

Right when predefined marks are open for the illuminating chronicles, new unlabeled depictions arrange into one of the predefined packs related with the names. Every now and then, by utilizing an outline set it tries to discover a depiction deal with which can be utilized to check or suspect new examination concerning the party. Thusly, indicate examination prescribes unsupervised strategy. However the term of enthusiasm with no restriction inside information mining suggest regulated gathering. [9] And besides, the 
articles Segmentation and Partitioning are utilized as an indistinct verbalization of gathering. The terms controlling at times propose diagram appropriated sub structure and division are utilized for separating information into get-together utilizing vital system, for example, gathering individuals in light of their remuneration.

Truly as Wikipedia references, the terms of K-derives gathering estimation was first made by J. MacQueen (1967) and after that the contemplating was trailed by J. A. Hartigan and M.A.Wong around 1975. The standard consider a system for beat code change was proposed the by Stuart Lloyd in 1957, notwithstanding it wasn't passed on until 1982.

The probability of K-assembles was showed up as before timetable as 1956 by Steinhaus [15]. A basic neighborhood heuristic for issue was proposed in 1957 by Lloyds [16]. The philosophy watches out for that disguised drive picking $\mathrm{k}$ discretionarily point as work environments. In each stage, present each point $\mathrm{X}$ into group with nearest office and a while later registers the purpose behind gathering of mass for every get-together. These centralizations of mass change into the new working environments for the running with sort out, and the system goes over until the point that the blueprint adjusts.

In part of how the neighbors are set up for each inside there exists some brilliant structures for performing K-infers:

- Lloyd's: Repeatedly applies Lloyd's estimation with subjectively took a stab at beginning stages.

- Swap: A territory search for heuristic, which works by performing swaps between existing fixations and a gameplan of contender focuses

This calculation iteratively changes focuses by performing swaps. Each run contains a given number (max swaps) executions of the swap heuristic.

\section{PROPOSED WORK AND RESULT}

The K-proposes figuring finds the predefined number of social events. In the advantageous condition, it is especially integral to find the measure of get-togethers for cloud dataset on the runtime. The settling of number of packs may activate low quality social gathering. The proposed rationale finds the measure of get-togethers on the continue running in setting of the social affair quality yield. This framework works for both the cases i.e. for proposed number of get-togethers early and besides obscure number of get-togethers. The client has the adaptability either to settle the measure of parties or by information the base number of packs required. In the past case it works same as K-proposes check. In the last case the figuring diagrams the new gatherings by building up the social gathering counter by one in every complement until the point that it fulfills the legitimacy of package quality edge. The balanced estimation is as indicated by the accompanying:Eq. 2

Eq.3

Data: k: number of packs (for dynamic social event display k=2) Fixed number of get-togethers = yes or no (Boolean).

D: an educational record containing $\mathrm{n}$ objects. Yield:

A game-plan of k get-togethers.

Method:

1. Discretionarily pick k objects from D as the key package focuses.

\section{Rehash.}

3. (re)assign every request the pack to which the test is most essentially indistinguishable, in light of the

mean estimation of the things in the social affair.

4. Strengthen the social affair construes, i.e. figure the mean estimation of the articles for each group.

5. until no change.

6. On the off chance that fixed_no_of_clusters =yes goto 11 .

7.Compute between bunch disconnect utilizing Eq.2

8.Compute intra-assemble remove utilizing

Eq. 3.

9.If new intra assemble remove < old_intra_cluster parceled and new_inter-

bundle >old_inter_cluster disconnect goto

10. else goto 11 .

11. $\mathrm{k}=\mathrm{k}+1$ goto compose 1 .

\section{STOP}

This diagram shows relationship of existing kmean tally and enhanced $\mathrm{k}$ mean calculation in light of number of package and number of cycle performed by estimations.

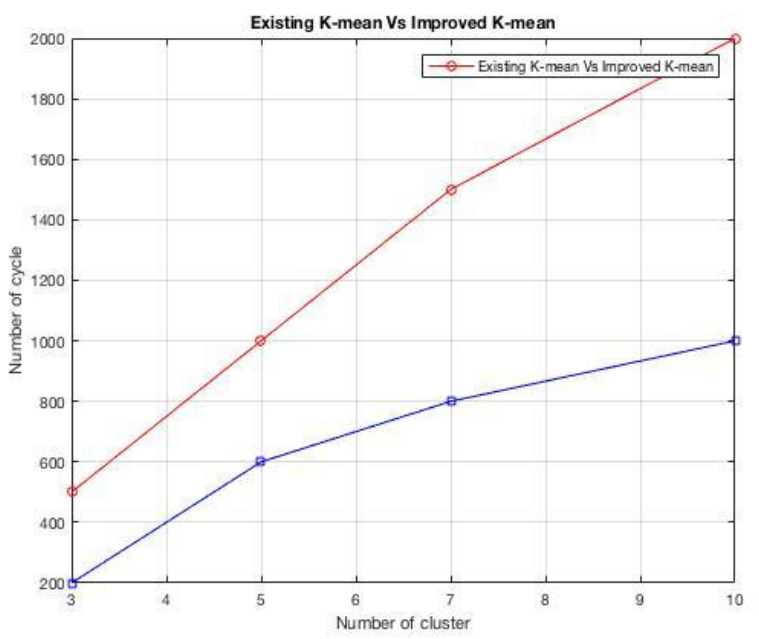

\section{CONCLUSION}

In this Paper, the issue was to manage the $\mathrm{K}$-gathering issue by exhibiting a get-together system - Multilevel setting of Kproposes. The issue in get-together as we find in result some piece of area 5, is a put off result of the method for K-derives technique which in the vital move the centroids are introduced unpredictably. Sometimes we have a poor gathering (a couple social affairs don't have any part). The objective is batching in the best lead, which ought to be to put away close information thinks however much as could sensibly be ordinary. In any case, with chief K-recommends gathering this rarly is the condition.

To streamline K-recommends, we propose a tally. Which in this structure at first take 2 information inspirations driving $\mathrm{N}$ information focuses subjectively were picked, after figure consistent of every this 2 server farms, impact one new information to point. By then we decrease the level of information demonstrate $N / 2$ and rehash this diminishment until the point that the moment that the measure of server 
develops in last reducing, are equivalent or more essential than $10 \%$ of N. By then we were running K-determines check of each layer and moreover in each layer, 10000 times by trading focuses among parties and get the base SSE, we try to reach to idealize get-together.

There are a couple of unmistakable approaches to manage administer widen our outcomes. Regardless; the present model can manage just a basic event of pivotal K-induces gathering. The issue of how to coordinate wide obliged Kproposes packaging still stays open. It legitimizes saying that in this estimation instead of picking discretionarily every two point, another technique for lessening can be utilized. It could be viewed as an examination consider in itself to discover a framework for picking these two concentration interests.

\section{REFERENCES}

[1] M. S. V. K. Pang-NingTan, "Data mining," in Introduction to data mining, Pearson International Edition, 2016, pp. 2-7.

[2] J.Peng and Y. Wei, "Approximating k-meanstype clustering via semi definite programming," SIAM Journal on Optimization, vol. 18, 2014.

[3] D.Alexander,"DataMining,"[Online].Available: http://www.laits.utexas.edu/ norman/BUS.FOR/course. mat/Alex/.

[4] "What is Data Repository," GeekInterview, 4 June 2013. [Online]. Available: http://www.learn.geekinterview.com/data-warehouse/dwbasics/what-is-data-repository.html.

[5] Fayyad, Usama; Gregory Piatetsky- Shapiro, and Padhraic Smyth (2016), from Data mining to knowledge discovery in data base

[6] M. S. V. K. Pang-NingTan, "Data mining," in Introduction to data mining, Pearson International Edition , 2015 pp. 8.
[7] M.S.V.K. Pang-NingTan, "Data mining," in Introduction to data mining, Pearson International Edition, 2016, pp. 7-11.

[8] Han, Jiawei, Kamber, Micheline. (2014) Data Mining: Concepts and Techniques. Morgan Kaufmann

[9] M. S. V. K. Pang-NingTan, "Data mining," in Introduction to data mining, Pearson International Edition , 2015, pp. 487-496.

[10] "An Introduction to Cluster Analysis for Data Mining," 2013. [Online]. Available: http://www.cs.umn.edu/ han/dmclass/cluster_survey_10 _02_00.

[11] Joaquín Pérez Ortega, Ma. Del Rocío Boone Rojas, María J. Somodevilla García Research issues on, $K$ means Algorithm: An Experimental Trial Using Matlab

[12] J. MacQueen, "Some Methods For Classification And Analysis Of Multivariate Observations," In proceedings of the Fifth Berkeley Symposium on Mathematical Statistics and Probability, University of California Press, 2015, pp. 281-297

[13] M. S. V. K. Pang-NingTan, "Data mining," in Introduction to data mining, Pearson International Edition , 2012, pp. 496-508

[14] Jain, A.K., Murty, N.M. and Flynn, P.J. (2015) Data Clustering: A Review.ACM Computing Surveys, Vol.31 No.3, pp. 264-323.

[15] V.Braverman,A.Meyerson,R.Ostrovsky, A. Roytman, M. Shindler and B. Tagiku, "Streaming k-means on WellClusterable Data," pp. 26-40, 2015.

[16] Stuart Lloyd. "Least Squares Quantization in PCM". In Special issue on quantization, IEEE Transactions on Information Theory, volume 28, PP. 129,137, 2014. 